Pacific Journal of Mathematics

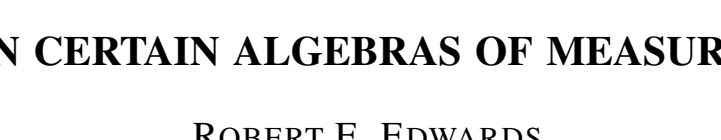




\title{
ON CERTAIN ALGEBRAS OF MEASURES
}

\author{
R. E. EDWARDS
}

1. Introduction and summary. Let $G$ be a group, assumed locally compact but not necessarily abelian. Elements of $G$ are denoted by $x, y, \cdots ; G$ will be written multiplicatively, and $e$ denotes the neutral element of $G . E$ is a vector space (under pointwise operations) of numerical functions $f=f(x)$ on $G$, and $F$ is a vector space of Radon measures on $G$. The following hypotheses are assumed to hold:

(I) Every $f \in E$ is integrable for every $\mu \in F$.

(II) $F$ is invariant under convolution.

(III) If $f \in E, \mu \in F$, and $g(x)=\int_{G} f(x y) d \mu(y)$, then $g \in E$.

(IV) $F$ is total over $E$.

As illustrations we mention

Example 1. E the vector space of all continuous numerical functions on $G ; F$ the set of all Radon measures on $G$ having compact supports.

Example 2. E the set of all bounded (or locally essentially bounded) Haarmeasurable functions on $G ; F$ the set of all bounded Radon measures absolutely continuous with respect to Haar measure. Strictly speaking, in order that (IV) be fulfilled we must reduce $E$ modulo functions which vanish locally a.e.

Example 3. Take $G=R$, the real line, $E$ the set of all continuous functions of polynomial order at infinity, and $F$ the set of all "rapidly decreasing" measures on $R$. A measure $\mu$ on $R$ is said to be "rapidly decreasing" if

$$
\int_{R}|x|^{k} d|\mu|(x)<+\infty
$$

for $k=0,1,2, \ldots$

Returning to the general situation, we agree to equip $F$ with the weak topology $\sigma(F, E)$ defined by the duality set up by the bilinear form

Received October 6, 1953.

Pacific J. Math. 5 (1955), 379-389 


$$
\langle f, \mu\rangle=\int_{G} f(x) d \mu(x)
$$

In view of (II), $F$ may also be regarded as an algebra with the ring product defined as convolution.

This note is concerned with two separate questions. First, it is a matter of experience that $F$ is rarely a genuine topological algebra in so far as the mapping $(\mu, \nu) \longrightarrow \mu * \nu$ of $F * F$ into $F$ generally fails to be continuous. These cases are investigated in Theorem 1 . In the second place, there is the question of the existence of maximal and primary ideals in $F$, and that of the analysis of a general closed ideal in $F$ in terms of these. No general results are forthcoming in this paper. We shall merely draw attention to some results which are given by L. Schwartz for the case of Example 1 with $G=R$ and extend these to the case of Example 3. This extension is given in Theorem 2. A few additional comments on this particular algebra of measures are given in $\S 4$.

2. Weak continuity of the convolution. The extreme rarity of cases in which continuity in the pair is attained is illustrated by

THE OREM 1. In order that the mapping $(\mu, \nu) \rightarrow \mu * \nu$ of $F \times F$ into $F$ be continuous for the topology $\sigma(F, E)$, it is necessary and sufficient that each $f \in E$ be a finite linear combination of coordinates of a finite-dimensional linear representation of $G$ which themselves belong to $E$.

Proof. We prove sufficiency first. Let $f \in E$ and suppose that there is a finite-dimensional linear representation $s \rightarrow M(s)$ of $G$ of degree $n$, say

$$
M(s)=\left\|m_{i j}(s)\right\|_{1 \leq i, j \leq n},
$$

for which scalars $c_{i j}$ exist such that

$$
f(x)=\sum_{i, j} c_{i j} m_{i j}(x)
$$

identically in $x$. In (2.1) we may suppose only those $m_{i j}$ retained which are linearly independent; and by hypothesis, these $m_{i j} \in E$. We have then

$$
f(x y)=\sum_{i, j} c_{i j} m_{i j}(x y)=\sum_{i, j} c_{i j} \sum_{k} m_{i k}(x) m_{k j}(y)=\sum_{i, j, k} c_{i j} m_{i k}(x) m_{k j}(y)
$$


identically in $x$ and $y$. If $\mu, \nu \in F$ one has by definition of $\mu * \nu$ :

$$
\langle f, \mu * \nu\rangle=\int_{G} \int_{G} f(x y) d \mu(x) d \nu(y)=\sum_{i, j, k} c_{i j} \cdot\left\langle m_{i k}, \mu\right\rangle \cdot\left\langle m_{k j}, \nu\right\rangle .
$$

Putting

$$
K=\frac{1}{n} \sum_{i, j}\left|c_{i j}\right|
$$

it follows that

$$
\left|\left\langle m_{i k}, \mu\right\rangle\right| \leq K^{-1} \text { and }\left|\left\langle m_{j k}, \nu\right\rangle\right| \leq K^{-1} \quad(1 \leq i, j, k \leq n)
$$

implies $|\langle t, \mu * \nu\rangle| \leq 1$, so that weak continuity is attained.

Turning now to the proof of necessity, let us write $N_{f}(\mu)=|\langle f, \mu\rangle|$ for $f \in E$ and $\mu \in F$. If convolution is continuous in the pair, given $f \in E$ one can find a finite family $\left(f_{i}\right)_{1 \leq i \leq n}$ of elements of $E$ such that, for $\mu$ and $\nu$ in $F$, the relations

$$
\sup _{1 \leq i \leq n} N_{f_{i}}(\mu) \leq 1 \text { and } \sup _{1 \leq i \leq n} N_{f_{i}}(\nu) \leq 1
$$

imply the relation

$$
N_{f}(\mu * \nu) \leq 1
$$

Now, by (IV), $F$ is separated for its weak topology. So, since one may plainly assume that the $f_{i}$ are linearly independent, measures $\phi_{i} \in F$ can be found such that

$$
\left\langle f_{j}, \phi_{i}\right\rangle=\delta_{i j} \quad(1 \leq i, j \leq n) .
$$

Because (2.2) implies (2.3), whatever $\mu$ and $\nu$ in $F$, the measure

$$
\left[\mu-\sum_{i=1}^{n}\left\langle f_{i}, \mu\right\rangle \phi_{i}\right] *\left[\nu-\sum_{j=1}^{n}\left\langle f_{j}, \nu\right\rangle \phi_{j}\right]
$$

is orthogonal to $f$. If this expression is expanded, appeal made to the FubiniTonelli theorem, and account taken of (IV), it results that $f$ satisfies the functional equation 


$$
f(x y)=\sum_{i=1}^{n}\left\{f_{i}(y) g_{i}(x)+f_{i}(x) g_{i}(y)\right\}-\sum_{i, j=1}^{n} f_{i}(x) f_{j}(y) \cdot\left\langle f, \phi_{i} * \phi_{j}\right\rangle,
$$

where

$$
g_{i}(x)=\int_{G} f(x y) d \phi_{i}(y)
$$

belongs to $E$ by (III). As a consequence, both the left and right translates of $f$ generate finite dimensional spaces. This, as it is very easy to see, implies that $f$ satisfies the condition stated in the theorem. The proof is thus complete.

REMARKS. (1) The hypotheses (I)-(IV) are clearly enough to ensure that $(\mu, \nu) \longrightarrow \mu * \nu$ is continuous in each argument separately.

(2) In many cases $E$ is equipped a priori with a locally convex topology and $F$ is a subset of the dual $E^{\prime}$ of $E$. In such a situation it is well known that there will usually be special subsets of $F$ the mapping $(\mu, \nu) \longrightarrow \mu * \nu$ restricted to these subsets will be continuous in each argument. A simple instance arises when $E$ is the Banach space of all continuous functions on $G$ which tend to zero at infinity, equipped with the norm

$$
\|f\|_{\infty}=\sup _{x \in G}|f(x)|,
$$

and $F$ is the dual of $E$ (the space of all bounded Radon measures on $G$ ). It is then easy to show that the convolution, qua function of two variable measures, is such that its restriction to each bounded subset of $F \times F$ is weakly continuous.

3. Analysis of ideals. Throughout the rest of this paper we attach to $E$ and $F$ the significance explained in Example 3 above. We recall that, for the situation described in Example 1 with $G=R$, L. Schwartz ( $[2], \S 20)$ has demonstrated the possibility of analysing all closed ideals in $F$ in terms of their cospectra. In this section we aim to do the same thing for Example 3, the method being based upon more recent work of Schwartz [3].

To begin with we observe that it is possible to construct explicitly locally convex topologies on $E$ relative to which $F$ is precisely the dual of $E$. We comment briefly on this in $\S 4$, but it is irrelevant at the present stage since the obvious such topology, namely $\sigma(E, F)$, serves our needs equally well.

For $\mu \in F$ we define the Fourier transform 


$$
\hat{\mu}(y)=\int_{R} \exp (2 \pi i y x) d \mu(x)=\langle\exp (2 \pi i y x), \mu\rangle
$$

for $y \in R$. It is plain that $\hat{\mu}$ is a function having bounded derivatives of all orders given by

$$
\hat{\mu}^{(m)}(y) \equiv\left(\frac{d}{d y}\right)^{m} \hat{\mu}(y)=\int_{R}(2 \pi i x)^{m} \exp (2 \pi i y x) d \mu(x)
$$

$$
=\left\langle(2 \pi i x)^{m} \exp (2 \pi i y x), \mu\right\rangle
$$

for $m=0,1,2, \ldots$. For any real $\alpha$ and any positive integer $m$, we denote by $M_{\alpha}^{(m)}$ the set of $\mu \in F$ such that $\hat{\mu}^{(p)}(\alpha)=0$ for $0 \leq p<m$. This definition is extended to the two extreme cases

$$
\begin{aligned}
& m=0: M_{a}^{(0)}=F \\
& m \neq \infty: M_{a}^{(\infty)}=\left\{\mu \in F: \hat{\mu}^{(p)}(\alpha)=0 \text { for } p=0,1,2, \cdots\right\} .
\end{aligned}
$$

We write $M_{\alpha}$ in place of $M_{a}^{(1)}$. Each $M_{\alpha}^{(m)}$ is an ideal in $F$, plainly closed; the $M_{\alpha}$ are the only closed maximal ideals in $F$, and the $M_{\alpha}^{(m)}$ with $m>0$ the only closed primary ideals.

We will now state and prove

THEOREM 2. If $I$ is any closed ideal in $F$, then

$$
I=\cap M_{\alpha}^{(m)}
$$

the intersection ranging over all the $M_{a}^{(m)}$ containing $l$.

Proof. By the Hahn-Banach theorem, an equivalent assertion is this: if $f \in E, f$ is the weak limit of linear combinations of exponential-monomials $(2 \pi i x)^{p} \exp (2 \pi i \alpha x)$ belonging to the weakly closed and translation-invariant vector subspace of $E$ generated by $f$. To see that this is indeed the case, we imbed $E$ in the space $\varnothing^{\prime}$ of temperate distributions over $R$ and use Theorème VI of [3]. This last tells us that the said approximation is possible in the sense of the topology of $\$$ : To complete the argument we apply the following lemma, which was suggested to the author by M. Schwartz.

Lemma. Let $H$ be a translation-invariant vector subspace of $E$. Then the 
weak closure of $H$ in $E$ is precisely the intersection of $E$ with the closure of $H$ in '. $^{\circ}$

Proof. Let $H_{1}$ and $H_{2}$ denote respectively the closures of $H_{1}^{\prime}$ in $E$ and $\$$ '. Trivially $H_{1} \subset H_{2}$ (since $\sigma(E, F)$ is finer than $\sigma\left(\wp^{\prime}, \bigotimes\right)$ ) and so also $H_{1} \subset$ $H_{2} \cap E$. To prove that conversely $H_{2} \cap E \subset H_{1}$, we argue by contradiction. If the assertion were false, there would be an $f_{0} \in\left(H_{2} \cap E\right) \cap C H_{1}$. That $f_{0} \in \mathrm{CH}_{1}$ would entail the existence of $\mu \in F$ such that $f * \mu=0$ for all $f \in H$ and $f_{0} * \mu \neq 0$. One could then choose a function $\phi$ with derivatives of all orders and with a compact support such that $f * \mu * \phi=0$ and $f_{0} * \mu * \phi \neq 0$. Since then $\mu * \phi \in \varnothing$, one would conclude that $f_{0} \in \mathrm{CH}_{2}$, the desired contradiction. This proves the lemma and, with it, the theorem also.

To make possible a more direct comparison with the results of [2], it is necessary to rewrite $(3.3)$ in the form

$$
I=\prod M_{a}^{m}
$$

where on the right one has products, rather than intersections, of ideals. The passage from (3.3) to $(3.4)$ is not completely trivial and we proceed to indicate how it may be effected.

Since the product of an infinite set of ideals is by definition the intersection of all finite partial products, the identity of the right members of (3.3) and (3.4) will follow once it is shown that

$$
M_{a_{1}}^{m_{1}} \ldots M_{a_{k}}^{m_{k}}=M_{a_{1}}^{\left(m_{1}\right)} \cap \ldots n M_{a_{k}}^{\left(m_{k}\right)}
$$

for any finite selection of real numbers $\alpha_{1}, \cdots, \alpha_{k}$ and of integers $m_{1}, \cdots, m_{k}$. The $m_{j}$ are, a priori, possibly infinite, but it is once again enough to deal with the case in which each $m_{j}$ is finite.

As a first step we will show that

$$
M_{a}^{(m)}=M_{a}^{m}
$$

for any (finite) integer $m$. This is trivial (by definition of each side) when $m=0$, so we may assume that $0<m<\infty$. On the one hand, if $\mu \in M_{a}^{m}, \mu$ is the limit of finite sums of measures $\nu_{1} * \ldots * \nu_{m}$ with each $\nu_{i} \in M_{a}$. Each such convolution has a Fourier transform which is divisible by $(y-\alpha)^{m}$; hence the same is true of any finite sum of such convolutions, and finally the same is 
true for any limit in $F$ of such finite sums since the convergence in $F$ implies, according to (3.2), the pointwise converge of the Fourier transform and of each of its derivatives. Thus it follows that $M_{a}^{m} \subset M_{\alpha}^{(m)}$. Conversely assume that $\hat{\mu}(y)$ is divisible by $(y-\alpha)^{m}$. Now consider the measures of the form

$$
\lambda=\left(\frac{1}{2 \pi i} \frac{d}{d x}-\alpha\right)^{m} \phi_{1} * \cdots * \phi_{m},
$$

where the $\phi_{i}$ range separately over all functions with derivatives of all orders and with compact supports. If $f \in E$ is orthogonal to all measures (3.7), then, since these form a translation-invariant set, $\hat{\lambda}(y) \hat{f}=0$ in the sense of distributions. This means that

$$
(y-\alpha)^{m} \hat{\phi}_{1}(y) \cdots \hat{\phi}_{m}(y) \cdot \hat{f}=0
$$

for all $\phi_{1}, \cdots, \phi_{m}$. Since $(y-\alpha)^{m}$ divides $\hat{\mu}(y)$, it follows easily that $\hat{\mu}(y)$. $\hat{f}=0$ also. By the Hahn-Banach theorem this shows that the finite sums of measures of the form $(3.7)$ are dense in $M_{\alpha}^{(m)}$. However, since we can write

$$
\lambda=\left[\left(\frac{1}{2 \pi i} \frac{d}{d x}-\alpha\right) \phi_{1}\right] * \ldots *\left[\left(\frac{1}{2 \pi i} \frac{d}{d x}-\alpha\right) \phi_{m}\right]
$$

with each square bracket on the right enclosing a member of $M_{\alpha}$ ( since it has a transform divisible by $(y-\alpha))$, it results that $M_{\alpha}^{m}$ is dense in $M_{\alpha}^{(m)}$. But $M_{a}^{m}$ is closed by definition. Hence $M_{a}^{m} \supset M_{a}^{(m)}$ and so the proof of $(3.6)$ is complete.

The proof of (3.5) may now be finished, starting from (3.6), by exactly the same methods as used in the proof of (3.6) itself.

We remark also that in the case dealt with by Schwartz in [2], the relevant $\alpha$ are generally complex and all the $m$ are forcibly finite; in the present case the $\alpha$ are forcibly real and the $m$ may be infinite. Further, the case dealt with by Schwartz has never been extended to more than one dimension; in the present case there appears to be no barrier in the path of such an extension, save perhaps increased complexity.

To close this section, we may observe that it may be proved quite easily that $M_{a}$ consists precisely of those $\mu \in F$ having a representation

$$
\mu=\left(\frac{1}{2 \pi i} \frac{d}{d x}-\alpha\right) \nu
$$


for a suitably chosen $\nu \in F$, the derivative being in the sense of distributions.

4. The duality between $E$ and $F$. At least a minor interest attaches itself to the determination of the finest topology $\tau(E, F)$ on $E$ compatible with the duality between $E$ and $F$, since this allows one to estimate just how strong will be the approximation theorems established by a direct application of duality theory. The determination of $\mathcal{T}(E, F)$ is equivalent, according to a theorem of Mackey-Arens, to that of characterising all the weakly compact and circled subsets of $F$.

A numerical sequence $\sigma=\left(\sigma_{n}\right)_{1}^{\infty}$ will be termed "rapidly decreasing" if $\lim _{n \rightarrow \infty} n^{k} \sigma_{n}=0$ for $k=0,1,2, \ldots$.

If $\mu$ is a bounded Radon measure, we define

$$
\|\mu\|=\sup \left|\int_{R} f d \mu\right|
$$

for $f$ continuous, having a compact support, and such that $\|f\|_{\infty} \leq 1$. If $A$ is a Borel set, the restriction $\mu_{A}$ of $\mu$ to $A$ is defined by

$$
\int_{R} f d \mu_{A}=\int_{A} f d \mu
$$

for $f$ continuous and with a compact support. If $A$ is open,

$$
\left\|\mu_{A}\right\|=\sup \left|\int_{R} f d \mu\right|
$$

for $f$ continuous, $\|f\|_{\infty} \leq 1$, and the support of $f$ being contained in $A$.

The set $\mathcal{K}$ of all continuous functions with compact supports forms a vector space, and it is known that $\mathcal{K}$ may be formed into an $(L F)$-space in such a way that its dual $Z^{\prime}$ is precisely the vector space of all Radon measures on $R$ see for example, Bourbaki [ 1, Exercise 1, p. 64].

The main theorem of this section is

THE OREM 3. Let $\Omega_{1}$ be a bounded open neighbourhood of $[-1,1]$ and let $\Omega_{n}(n \geq 2)$ be a bounded open neighbourhood of the set of real $x$ satisfying $n-1 \leq|x| \leq n$. For any rapidly decreasing sequence $\sigma=\left(\sigma_{n}\right)$, let $M_{\sigma}$ denote the set of measures $\mu \in F$ satisfying the system of inequalities

$$
\left\|\mu_{\Omega_{n}}\right\| \leq \sigma_{n} \quad(n=1,2, \cdots) .
$$


As $\sigma$ varies, the $M_{\sigma}$ form a base for the set of weakly compact subsets of $F$.

An almost immediate corollary is the following.

Corolla Ry. The topology $\tau(E, F)$ is that defined by the seminorms

$$
p_{\sigma}(f)=\sum_{n=1}^{\infty} \sigma_{n} \cdot \sup _{n-1 \leq|x| \leq n}|f(x)| \quad(f \in E),
$$

with $\sigma=\left(\sigma_{n}\right)$ ranging over all rapidly decreasing sequences.

For the proof we shall require the

LEMMA. If $M \subset Z^{\prime}$ is such that

$$
\sup _{\mu \in M}\left\|\mu_{\Omega_{n}}\right\|<+\infty \quad(n=1,2, \cdots),
$$

$\left(\Omega_{n}\right)$ being any sequence of bounded open sets covering $R$, then $M$ is weakly relatively compact in $\mathfrak{k}$.

Proof. Let $\rho_{n}>0$ be defined by

$$
\frac{1}{\rho_{n}}=\sup _{\mu \in M}\left\|\mu_{\Omega_{n}}\right\| \text {. }
$$

Then $M$ is contained in the polar set $U^{o}$ in $Z^{\prime}$ of the set $U \subset \mathcal{Z}$ defined by

$$
U=\left\{f \in \mathcal{K}: \text { support of } f \subset \Omega_{n} \text {, and }\|f\|_{\infty} \leq \rho_{n}\right\} \text {. }
$$

This set $U$ is a neighbourhood of 0 in the $(L F)$-space $\mathcal{Z}$ and so, by a general theorem, $U^{o}$ is weakly compact in $\mathcal{K}$ :

Proof of Theorem 3. First we will show that every set of the form $M_{\sigma}$ is weakly compact in $F$. For this we must show that if $\sigma$ is given rapidly decreasing, and if $\Phi$ is any filter on $M_{\sigma}$, then there is a filter $\Phi^{\prime}$, finer than $\Phi$, and a measure $\nu \in M_{\sigma}$ such that $\Phi^{\prime}$ converges weakly to $\nu$. Since $M_{\sigma}$ is plainly weakly closed in $F$, it is enough to produce a $\nu \in F$ with the said properties.

Now by the lemma, there is a filter $\Phi^{\prime}$ finer than $\Phi$ and a measure $\nu \in \mathcal{Z}$ ' such that $\Phi^{\prime}$ converges to $\nu$ weakly in $\chi^{\prime}$, that is,

$$
\lim _{\mu \in \Phi^{\prime}} \int_{R} f d \mu=\int_{R} f d \nu
$$


for each $f \in \mathcal{K}$. This implies already that $\left\|\nu_{\Omega_{n}}\right\| \leq \sigma_{n}$ and hence that $\nu \in F$. It remains to show that $\Phi^{\prime}$ converges to $\nu$ weakly in $F$.

Let $f \in E$ be given and fix an integer $k$ so that

$$
a=\sup _{x \in R}(1+|x|)^{-k}|f(x)|<+\infty
$$

Clearly, for each $n$ one can find a function $f_{n} \in \mathcal{K}$, coinciding with $f$ on $\bigcup_{r=1}^{n} \Omega_{r}$, and such that

$$
(1+|x|)^{-k}\left|f(x)-f_{n}(x)\right| \leq a
$$

everywhere. Then, if $\mu \in M_{\sigma}$ one has

$$
\left|\int_{R} f d \mu-\int_{R} f_{n} d \mu\right| \leq \sum_{m>n} \int_{\Omega_{m}}\left|f-f_{n}\right| d|\mu| \leq a \sum_{m>n}(1+m)^{k} \sigma_{m},
$$

which tends to 0 as $n \longrightarrow \infty$. Thus, given $\epsilon>0$, one can find $n=n(\epsilon)$ such that, uniformly for $\mu \in M_{\sigma}$,

$$
\left|\int_{R} f d \mu-\int_{R} f_{n} d \mu\right| \leq \epsilon
$$

There is a set $A$ of the filter $\Phi^{\prime}$ such that $\mu \in A$ implies

$$
\left|\int_{R} f_{n} d \mu-\int_{K} f_{n} d \nu\right| \leq \epsilon
$$

Hence for $\mu \in A$.

$$
\left|\int_{R} f d \mu-\int_{R} f d \nu\right| \leq 3 \epsilon
$$

Since $\epsilon$ is arbitrary, this shows that $\Phi^{\prime}$ converges to $\nu$ weakly in $F$ and thus completes the proof that $M_{\sigma}$ is weakly compact in $F$.

To finish the proof of the theorem it is required to show that if $M \subset F$ is weakly compact, then $M \subset M_{\sigma}$ for some rapidly decreasing sequence $\sigma$. We will in fact show that this follows already from the apparently weaker hypothesis that $M$ is merely weakly bounded in $F$. For, let $E_{k}$ be the subspace of $E$ formed of those $f$ for which 


$$
N_{k}(f) \equiv \sup _{x \in R}(1+|x|)^{-k}|f(x)|
$$

is finite, $k$ being an integer $\geq 0 . E_{k}$, equipped with the norm $N_{k}$, is a Banach space. If $M$ is weakly bounded in $F$, it is a fortiori weakly bounded in the dual of $E_{k}$ for each $k$. Thus for each $k$ there is a finite $m_{k}$ such that

$$
\int_{R}(1+|x|)^{k} d|\mu|(x) \leq m_{k}
$$

for all $\mu \in M$. A fortiori, for each $n$ :

$$
n^{k}\left\|\mu_{\Omega_{n}}\right\| \leq m_{k}
$$

Thus, if $\sigma_{n}=\sup _{\mu \in M}\left\|\mu_{\Omega_{n}}\right\|$, then $n^{k} \sigma_{n} \leq m_{k}$ for $n, k=1,2, \ldots$. This shows that $\sigma=\left(\sigma_{n}\right)$ is rapidly decreasing and that $M \subset M_{\sigma}$.

\section{REFERENCES}

1. N. Bourbaki, Intégration, Ch. I-IV, Actualités Scientifiques et Industrielles 1175, Hermann (Paris) 1952.

2. L. Schwartz, Theórie générale des fonctions moyenne-périodiques, Ann. of Math. 48 (4) 1947, 859- 929.

3. - Analyse et synthèse harmoniques dans les espaces de distributions, Canadian J. Math. 3 (4), 1951, 503 - 512.

UNIVERSITY OF NANCY

NANCY, FRAACE 



\section{PACIFIC JOURNAL OF MATHEMATICS}

\section{EDITORS}

\author{
H.L. ROYDEN \\ Stanford University \\ Stanford, California \\ E. HEWITT \\ University of Washington \\ Seattle 5, Washington
}

\author{
R. P. DILWORTH \\ California Institute of Technology \\ Pasadena 4, California \\ * Alfred Horn \\ University of California \\ Los Angeles 24, California
}

\section{ASSOCIATE EDITORS}

\section{H, BUSEMANN \\ HERBERT FEDERER}

MARSHALL HALL

\section{P.R. HALMOS \\ HEINZ HOPF}

ALFRED HORN

\author{
R.D. JAMES \\ BØRGE JESSEN \\ PAUL LÉVY
}

GEORGE PÓLYA

J.J. STOKER

KOSAKU YOSIDA

\section{SPONSORS}

UNIVERSITY OF BRITISH COLUMBIA

UNIVERSITY OF SOUTHERN CALIFORNIA

CALIFORNIA INSTITUTE OF TECHNOLOGY

UNIVERSITY OF CALIFORNIA, BER KELEY

STANFORD RESEARCH INSTITUTE

STANFORD UNIVERSITY

UNIVERSITY OF CALIFORNIA, DAVIS

UNIVERSITY OF. UTAH

UNIVERSITY OF CALIFORNIA, LOS ANGELES

WASHINGTON STATE COLLEGE

UNIVERSITY OF CALIFORNIA, SANTA BARBARA

UNIVERSITY OF WASHINGTON

MONTANA STATE UNIVERSITY

UNIVERSITY OF NEVADA

OREGON STATE COLLEGE

AMERICAN MATHEMATICAL SOCIETY

HUGHES AIRCRAFT COMPANY

UNIVERSITY OF OREGON

SHELL DEVELOPMENT COMPANY

\section{UNIVERSTTY OF SOUTHERN CALIFORNIA}

Mathematical papers intended for publication in the Pacific Journal of Mathematics should be typewritten (double spaced), and the author should keep a complete copy. Manuscripts may be sent to any of the editors. Manuscripts intended for the outgoing editors should be sent to their successors. All other communications to the editors should be addressed to the managing editor, Alfred Horn, at the University of California Los Angeles 24, California.

50 reprints of each article are furnished free of charge; additional copies may be obtained at cost in multiples of 50 .

The Pacific Journal of Mathematics is published quarterly, in March, June, September, and December. The price per volume (4 numbers) is $\$ 12.00$; single issues, $\$ 3.50$; back numbers (Volumes $1,2,3$ ) are available at $\$ 2.50$ per copy. Special price to individual faculty members of supporting institutions and to individual members of the American Mathematical Society: $\$ 4.00$ per volume; single issues, $\$ 1.25$.

Subscriptions, orders for back numbers, and changes of address should be sent to the publishers, University of California Press, Berkeley 4, California.

Printed at Ann Arbor, Michigan. Entered as second class matter at the Post Office, Berkeley, California.

* During the absence of E.G. Straus.

UNIVERSITY OF CALIFORNIA PRESS - BERKELEY AND LOS ANGELES 


\section{Pacific Journal of Mathematics}

\section{Vol. 5, No. $3 \quad$ November, 1955}

Nesmith Cornett Ankeny and S. Chowla, On the divisibility of the class number of quadratic fields ............................. 321

Cecil Edmund Burgess, Collections and sequences of continua in the plane ........................................ 325

Jane Smiley Cronin Scanlon, The Dirichlet problem for nonlinear elliptic equations....................................... 335

Arieh Dvoretzky, A converse of Helly's theorem on convex sets ......... 345

Branko Grünbaum, On a theorem of L. A. Santaló................ 351

Moshe Shimrat, Simple proof of a theorem of P. Kirchberger .......... 361

Michael Oser Rabin, A note on Helly's theorem . ................... 363

Robert E. Edwards, On factor functions . . ................... 367

Robert E. Edwards, On certain algebras of measures ............... 379

Harley M. Flanders, Methods in affine connection theory.............. 391

Alfred Huber, The reflection principle for polyharmonic functions ........ 433

Geoffrey Stuart Stephen Ludford, Generalised Riemann invariants ....... 441

Ralph Gordon Selfridge, Generalized Walsh transforms............. 451 